\title{
Correlation Between Unidentified Bright Objects on Brain Magnetic Resonance Imaging (MRI) and Cerebral Glucose Metabolism in Patients with Neurofibromatosis Type 1
}

Young Bae Sohn', Young Sil An², Su Jin Lee ${ }^{2}$, Jin Wook Choi ${ }^{3}$, Seon-Yong Jeong', Hyon-Ju Kim ${ }^{1}$ and Jung Min Ko ${ }^{4}$

'Department of Medical Genetics, ${ }^{2}$ Department of Nuclear Medicine, ${ }^{3}$ Department of Radiology, Ajou University Hospital, Ajou University School of Medicine, Suwon, Kore, ${ }^{4}$ Department of pediatrics, Seoul National University Hospital, Seoul, Korea

\begin{abstract}
Purpose: Neurofibromatosis type 1 (NF1), which is caused by mutations of the NF1 gene, is the most frequent single gene disorder to affect the nervous system. Unidentified bright objects (UBOs) are commonly observed on brain magnetic resonance imaging (MRI) in patients with NF1. However, their clinical and pathologic significance is not well understood. The purpose of this study was to investigate the correlation between UBOs and cerebral glucose metabolism measured by ${ }^{18} \mathrm{~F}-2-$ Fluoro-2-deoxy-D-glucose $\left({ }^{18} \mathrm{~F}\right.$-FDG) positron emission tomography (PET) in Korean patients with NF1.

Materials and Methods: Medical records of 75 patients (34 males and 41 females) with NF1 who underwent brain MRI and PET between 2005 and 2011 were evaluated retrospectively. Clinical data including demographics, neurological symptoms, and brain MRI and PET findings, were reviewed.

Results: UBOs were detected in the brain MRI scans of 31 patients (41\%). The region most frequently affected by UBOs was the basal ganglia. The most frequent brain PET finding was thalamic glucose hypometabolism (45/75, 60\%). Of the 31 patients with UBOs, 26 had thalamic glucose hypometabolism on brain PET, but the other 5 had normal brain PET findings. Conversely, of the 45 patients with thalamic glucose hypometabolism on brain PET, 26 showed UBOs on their brain MRI scans, but 19 had normal findings on brain MRI scans.

Conclusion: UBOs on brain MRI scans and thalamic glucose hypometabolism on PET appear to be 2 distinctive features of NF1 rather than correlated symptoms. Because the clinical significance of these abnormal imaging findings remains unclear, a longitudinal follow-up study of changes in clinical manifestations and imaging findings is necessary.
\end{abstract}

Key words: Neurofibromatosis 1, Unidentified bright objects, Positron emission tomography, Cerebral glucose metabolism

\section{Introduction}

Neurofibromatosis type I (NF1) is the most common autosomal dominant neurocutaneous syndrome affecting approximately
1 in every 3,500 individuals. ${ }^{1,2)}$ Clinical symptoms of NF1 include multiple café au lait spots, neurofibromas in the skin, optic nerve glioma, Lisch nodules on the cornea, axillary or inguinal freckling, and skeletal dysplasia.

Received: 22 Nov 2012, Revised: 13 Dec 2012, Accepted: 18 Dec 2012, Published: 31 Dec 2012

${ }^{*}$ Corresponding author: Jung Min Ko, M.D.

Department of Pediatrics, Seoul National University Children's Hospital, 101 Daehak-ro, Jongno-gu, Seoul 110-769, Korea

Tel: +82-2-2072-3570, Fax: +82-2-743-3455, E-mail: jmko@snu.ac.kr

(c) This is an open-access article distributed under the terms of the Creative Commons Attribution Non-Commercial License (http://creativecommons.org/licenses/by-nc/3.0/) which permits unrestricted non-commercial use, distribution, and reproduction in any medium, provided the original work is properly cited. 
Focal areas of high signal intensity on T2-weighted images, which are defined as unidentified bright objects (UBOs), are commonly (43-93\%) observed on brain magnetic resonance imaging (MRI) scans of patients with $\mathrm{NF1}^{3,4)}$ The most commonly involved anatomic sites are the basal ganglia, cerebellum, midbrain, and the subcortical white matter. ${ }^{4)}$ However, UBOs are not always correlated with neurological symptoms, and are even present in patients without neurological symptoms. Pathologically, UBOs may represent increased fluid within myelin associated with hyperplastic or dysplastic glial proliferation. ${ }^{5}$ Although there have been several reports showing an association between UBOs and various clinical manifestations of NF1, including Lisch nodules, subcutaneous neurofibromas, optic glioma, other central nervous system neoplasms, cognitive deficits, and learning disabilities, ${ }_{1}^{6-8)}$ the clinical implications and functional characteristics of UBOs are not well understood.

Positron emission tomography (PET) with ${ }^{18} \mathrm{~F}$-2-Fluoro-2deoxy-D-glucose ( $\left.{ }^{18} \mathrm{~F}-\mathrm{FDG}\right)$ enables assessment of local cerebral glucose metabolism. The cerebral glucose metabolic rate is closely related to brain activity. In some patients with neurological abnormalities, the presence of areas of abnormal metabolism identified by PET has been attributed to cytoarchitectural disorders and biochemical abnormalities undetectable by conventional imaging techniques. ${ }^{10)}$ Therefore, ${ }^{18}$ F-FDG PET appears to be an appropriate tool for investigating putative functional correlations in the brains of patients with NF1.

The authors thus performed a retrospective review of patients with NF1, who underwent both brain MRI and ${ }^{18} \mathrm{~F}-\mathrm{FDG}$ PET, to investigate the functional characteristics of UBOs and to determine whether there is any correlation between UBOs and cerebral glucose metabolism.

\section{Materials and Methods}

Medical records of 75 patients (34 males and 41 females) diagnosed with NF1 based on standard clinical criteria ${ }^{8)}$ and who underwent brain MRI and PET between Jan 2005 and Dec 2011 were evaluated retrospectively. Patients who had intracranial tumors or who underwent cranial surgery were excluded. Clinical data including demographics, neurological symptoms, and brain MRI and PET findings, were reviewed. This study was approved by the institutional review board of Ajou University Hospital, Suwon, Korea (AJIRB-MED-MDB-12-344).

\section{MR imaging}

All MRI examinations were performed using a 1.5T MR imaging scanner (Genesis Signa, Signa Excite; GE Medical Systems, Milwaukee, WI) with a quadrature head coil. Unenhanced T1weighted (TE 9, TR 400), T2-weighted (TE 107, TR 3300), fluidattenuated inversion recovery (FLAIR; TE 142, TR 8800, TI 2200), and contrast-enhanced T1-weighted (TE 9, TR 400) images were obtained using axial spin echo sequences. Other imaging parameters were as follows: matrix $320 \times 256$; field of view, 20 $\mathrm{cm}$; slice thickness, $5 \mathrm{~mm}$; number of acquisitions, 1 .

\section{2. ${ }^{18}$ F-FDG PET studies}

PET/computed tomography (CT) data were acquired with a Discovery ST scanner (General Electric Medical Systems, USA). After fasting for at least $4 \mathrm{~h}$, patients were administered 300 $\mathrm{MBq}$ of ${ }^{18} \mathrm{~F}-\mathrm{FDG}$ intravenously. The serum glucose levels of all subjects were checked prior to ${ }^{18} \mathrm{~F}$-FDG injection. All were instructed to rest comfortably for $30 \mathrm{~min}$ with their eyes closed before image acquisition was initiated. We first performed a CT scan (tube-rotation time $1 \mathrm{~s}$ per revolution, 120 kV, 70 mA, 5.0 $\mathrm{mm}$ per rotation, acquisition time $11.8 \mathrm{~s}$, scan length $150.42 \mathrm{~mm}$ ). Thereafter, emission PET data were acquired at 8 min per frame in the three-dimensional mode. PET images were obtained by iterative reconstruction (i.e., ordered subsets of expectation maximization, with 1 iteration and 32 subsets), and the CT images were used to correct the attenuation.

\section{Statistical analysis}

All results are expressed as mean \pm standard deviation. A $P$ value $<0.05$ was considered significant. All statistical analyses were performed using SPSS version 15.0 (SPSS Inc., Chicago, IL, USA).

\section{Results}

The medical records of 75 patients with NF1 (34 males and 41 females) were reviewed. The mean age of the patients was $23.1 \pm$ 14.2 years; 32 patients were aged $<18$ years and 43 patients were aged $\geq 18$ years.

UBOs were apparent in the brain MRI scans of 31 patients (31/75, 41\%) (Fig. 1). Of these 31 patients, 20 were younger than 18 years. UBOs were most frequently observed in the basal ganglia (18/31, 

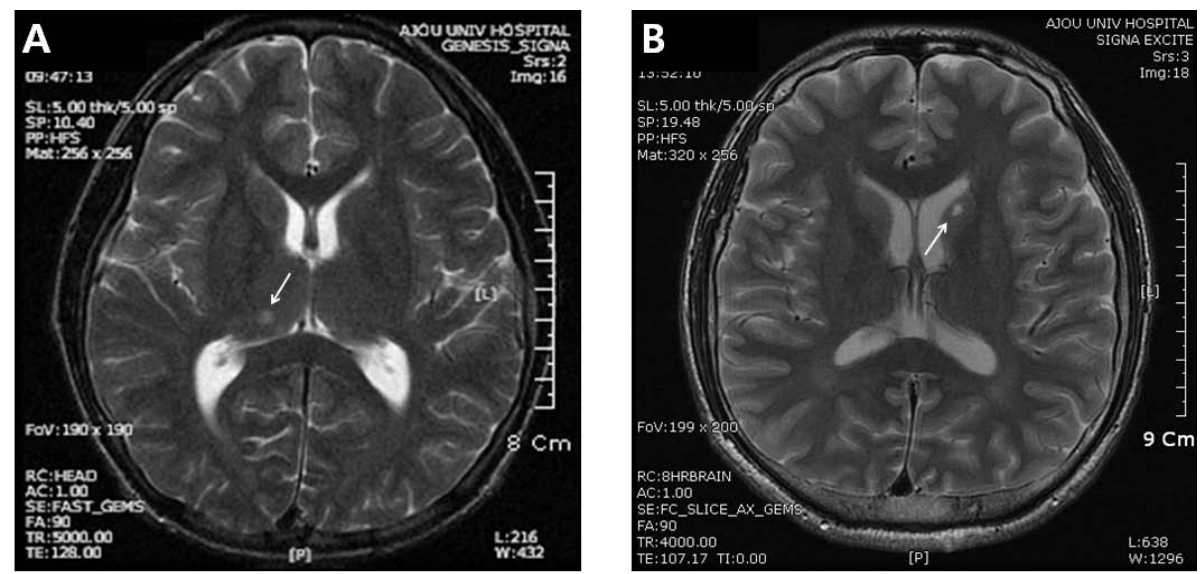

Fig. 1. Representative brain magnetic resonance imaging (MRI) showing unidentified bright objects (UBOs) (arrows) in the basal ganglia (A) and thalamus (B).

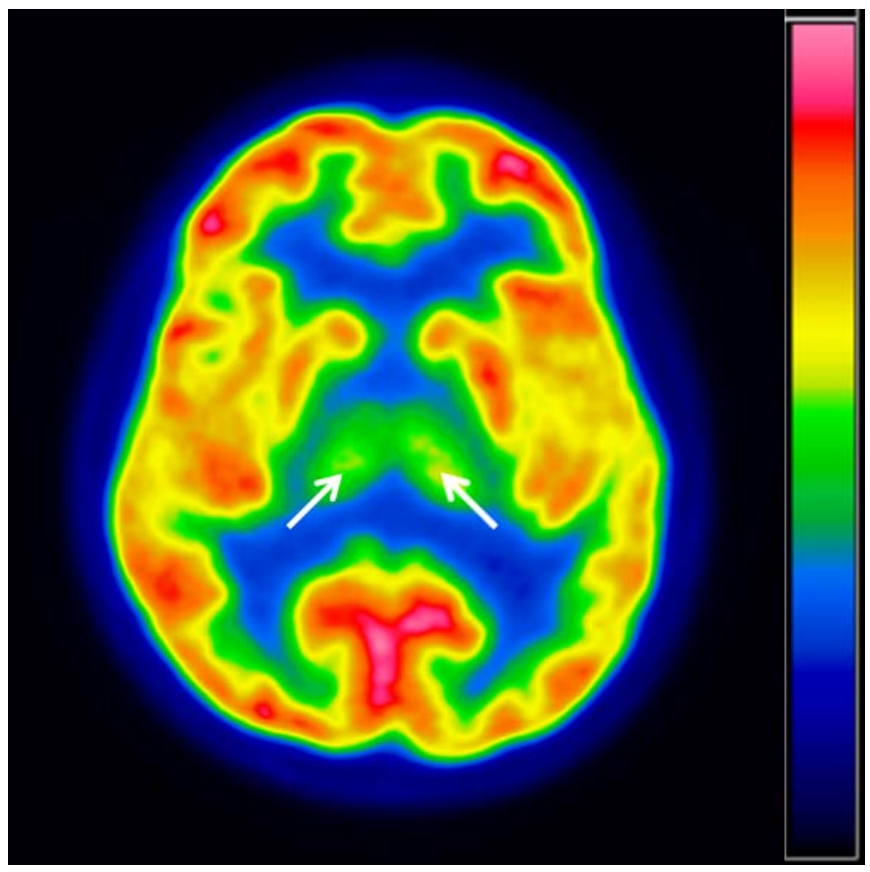

Fig. 2. Representative brain PET imaging showing reduced ${ }^{18} \mathrm{~F}-\mathrm{FDG}$ uptake in the bilateral thalamus presented in green color (arrows) that implicates bilateral thalamic glucose hypometabolism.

$58 \%)$, followed by in the cerebellum (16/31, 51\%), hippocampus $(12 / 31,39 \%)$, thalamus $(7 / 31,23 \%)$, pons $(3 / 31,10 \%)$, midbrain $(2 / 31,6 \%)$, and medulla oblongata $(2 / 31,6 \%)$.

Because patients who had an intracranial tumor or who had undergone cranial surgery were excluded from thisstudy, the only abnormal finding on brain PET was thalamic glucose hypometabolism presented in green color in brain PET imaging, which was detected in 45 patients (60\%). Reduced thalamic ${ }^{18} \mathrm{~F}-\mathrm{FDG}$ uptake was observed bilaterally (Fig. 2). The other 30 patients had normal brain PET finding implicating normal cerebral glucose metabolism.
Table 1. Number of Patients with or without UBO and Brain PET Abnormality

\begin{tabular}{lcc} 
& $\begin{array}{c}\text { Patients with } \\
\text { UBOs } \\
(n=31)\end{array}$ & $\begin{array}{c}\text { Patients without } \\
\text { UBOs } \\
(n=44)\end{array}$ \\
\hline $\begin{array}{l}\text { Thalamic glucose hypometabolism } \\
\text { on PET }(n=45)\end{array}$ & 26 & 19 \\
Normal brain PET $(n=30)$ & 5 & 25 \\
\hline
\end{tabular}

Of the 31 patients with UBOs, 26 (83.9\%) had thalamic glucose hypometabolism on brain PET, but the remaining 5 (16.1\%) patients had normal brain PET findings. Conversely, of the 45 patients with thalamic glucose hypometabolism detected by brain PET, 26 (57.8\%) had UBOs on their brain MRI scans. However, the other 19 (42.2\%) patients with thalamic glucose hypometabolism had normal brain MRI findings (Table 1).

We also analyzed 7 patients (2 males and 5 females) who had thalamic UBOs. Their median age was 7.2 (range, 4-26) years. None of them had focal neurologic symptoms. Thalamic glucose hypometabolism was identified by PET in 5 of these patients; the other 2 patients had normal brain PET images. Four of the patients completed a neuropsychiatric test. Their median Full-Scale I0 (FSIO) was 86.5 (76-98) as measured by the Korean Wechsler Intelligence Scale for Children 3rd edition (K-WISC-III) or the Korean Wechsler Adult Intelligence Scale (K-WAIS), whichever was most appropriate for their age. Their median social quotient (SO) as measured by social maturityscale (SMS) was 94.5 (81-118).

\section{Discussion}

UBOs are frequently found in the T2-weighted MR images of patients with NF1. However, the biochemical and functional 
characteristics of these UBOs remain unclear. Over the past 10 years, several groups have reported that mutation of the NF1 gene leads to multiple disorders of glial cell types, including astrocytes, oligodendrocytes, and microglia. ${ }^{11-14)}$ Although this could contribute to cerebral tissue damage, the biochemical and functional characteristics of such damage are not well understood.

In this study, 41\% of all patients had UBOs, which is consistent with the findings of previous studies that have not considered patient age. ${ }^{3,4)}$ Of the 31 patients with UBOs that were included in this study, 20 (65\%) were younger than 18 years. Twenty (60\%) of the 33 patients aged under 18 years had UBOs, whereas 11 (26\%) of the 42 patients aged 18 years and above had a UBO. The natural tendency of UBOs to disappear in adulthood is well known, ${ }_{1}^{14)}$ and seems to be consistent with our findings. However, despite our hypothesis that cerebral glucose metabolism could be helpful for providing information on the functional characteristics of UBOS, the presence of UBOs and cerebral glucose metabolism were not directly related. Moreover, these were also observed in patients with thalamic UBOs.

Moore et al. demonstrated a significant association between thalamic UBOs and deficits in I0, memory, motor function, distractibility, and attention domains, but no such associations were detected for UBOs in the basal ganglia, brain stem, or cerebellum. ${ }^{15)}$ The authors suggested that the simple presence or absence of UBOs, or their total number, is not as important as their location: UBOs in the cerebral cortex, basal ganglia, brain stem, or cerebellum seem to have no impact on neuropsychological function whereas UBOs in the thalamus do. Goh et al. also reported that UBOs in the thalamus were associated with lower intellectual function, and the size of the thalamic lesions was correlated with cognitive function. ${ }^{16)}$ In this study, 7 patients had thalamic UBOs. Of these, 5 (71.4\%) had thalamic glucose hypometabolism, and the other 2 (28.6\%) patients had normal PET images. Therefore, we could not find any direct associations between the presence of UBOs in the thalamus and glucose hypometabolism, but the number of subjects is too small to draw any definite conclusions.

The thalamus appears to be affected in patients with NF1, as shown by the reduction in scaled ${ }^{18} \mathrm{~F}-\mathrm{FDG}$ retention in their cerebral PET images. A hypometabolic pattern has been reported in several studies of brain PET images of patients with NF1. ${ }^{9}{ }^{10}$ ) Therefore, it is possible that there is a relationship between cerebral hypometabolism and cognitive impairment in patients with NF1. ${ }^{10)}$ North et al. suggested that the pathogenesis of the neurological deficit in patients with NF1 was as follows: mutations of the NF1 gene result in lesions in the CNS with aberrant control of cell growth and differentiation. ${ }^{6,17)}$ These lesions disrupt important neuronal circuits involved in higher cognitive processing and manifest as specific cognitive deficits rather than as focal neurological signs. Since the thalamus plays a significant role in motor coordination and in the integration of complex cognitive functions, thalamic dysfunction caused by the mechanism, as suggested by North et al., might indeed give rise to the observed NF1-related neurological deficits. ${ }^{9}$ In this study, in terms of cognitive function, 4 of the 7 patients with thalamic UBOs, who completed a neuropsychiatric test had a median FSIO of 86.5 (76-98), which is classified as "low average," and a median S0 of 94.5 (81-118), which is classified as "average." These results are thus ambiguous and it is not possible to draw any conclusions.

There are several limitations to this study. First, our data are cross-sectional, and a longitudinal follow-up study is needed because NF1 is a progressive disease. Although we excluded patients with brain tumors, Griffith et al. performed a longitudinal MRI study and reported that brain tumors in several children with NF1 developed at the sites of previously detected UBOs. ${ }^{3)}$ In addition, they reported that the children who developed brain tumors tended to have more UBOs than other children their age with NF1. ${ }^{3)}$ Therefore, a longitudinal study with follow-up brain imaging is warranted to investigate tumor development in patients with UBOs. Second, cognitive function was not analyzed and so cannot be correlated with the imaging abnormalities (no thorough neuropsychiatric evaluations were performed). Because this study was based on a retrospective chart review, the neuropsychiatric evaluations that were performed were inconsistent, and so it was practically impossible to obtain reliable data. In addition, no reliable statements about the relevance of the UBOs and thalamic glucose hypometabolism can be made since the brain MR findings and cerebral PET images appeared not to be related.

Balestri et al. previously suggested that UBOs and hypometabolism in brain PET images were independent and distinctive features of NF1 because metabolic activity was normal in areas that appeared to be abnormal on MRI. ${ }^{10)}$ Our study findings are consistent with their report, and we reproduced their findings in a relatively large number of subjects ( 75 patients). To further clarify the role of UBOs and thalamic glucose hypometabolism in NF1, a prospective longitudinal study with a larger number of subjects and with systemic assessment of neuropsychological function is required. 


\section{References}

1. Huson SM, Harper PS, Compston DA. Von Recklinghausen neurofibromatosis. A clinical and population study in south-east Wales. Brain 1988;111:1355-81.

2. Moharir M, London $K_{1}$ Howman-Giles $R$, North $K$. Utility of positron emission tomography for tumour surveillance in children with neurofibromatosis type 1. Eur J Nucl Med Mol Imaging 2010;37:1309-17.

3. Griffiths PD, Blaser S, Mukonoweshuro W, Armstrong D, MiloMason G, Cheung S. Neurofibromatosis bright objects in children with neurofibromatosis type 1: a proliferative potential? Pediatrics 1999;104:e49.

4. Margari L, Presicci A, Ventura P, Maria Bacca S, lliceto G, Medicamento $\mathrm{N}$, et al. Clinical and instrumental (magnetic resonance imaging [MRI] and multimodal evoked potentials) follow-up of brain lesions in three young patients with neurofibromatosis 1. J Child Neurol 2006;21:108590.

5. DiPaolo DP, Zimmerman RA, Rorke LB, Zackai EH, Bilaniuk LT, Yachnis AT. Neurofibromatosis type 1: pathologic substrate of high-signalintensity foci in the brain. Radiology 1995;195:721-4.

6. North KN, Riccardi V, Samango-Sprouse C, Ferner R, Moore B, Legius $E_{1}$ et al. Cognitive function and academic performance in neurofibromatosis. 1: consensus statement from the NF1 Cognitive Disorders Task Force. Neurology 1997:48:1121-7.

7. Szudek J, Friedman JM. Unidentified bright objects associated with features of neurofibromatosis 1. Pediatr Neurol 2002;27:123-7.

8. North $\mathrm{K}$, Hyman S, Barton B. Cognitive deficits in neurofibromatosis 1.J Child Neurol 2002;17:605-12; discussion 27-9, 46-51.

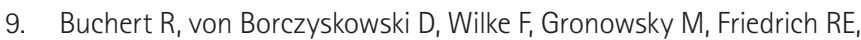
Brenner $W_{1}$ et al. Reduced thalamic 18F-flurodeoxyglucose retention in adults with neurofibromatosis type 1. Nucl Med Commun 2008;29:1726.

10. Balestri P, Lucignani G, Fois A, Magliani L, Calistri L, Grana C, et al. Cerebral glucose metabolism in neurofibromatosis type 1 assessed with [18F]-2-fluoro-2-deoxy-D-glucose and PET. J Neurol Neurosurg Psychiatry 1994;57:1479-83.

11. Bennett MR, Rizvi TA, Karyala S, McKinnon RD, Ratner N. Aberrant growth and differentiation of oligodendrocyte progenitors in neurofibromatosis type 1 mutants. J Neurosci 2003;23:7207-17.

12. Daginakatte GC, Gianino SM, Zhao NW, Parsadanian AS, Gutmann $\mathrm{DH}$. Increased c-Jun-NH2-kinase signaling in neurofibromatosis-1 heterozygous microglia drives microglia activation and promotes optic glioma proliferation. Cancer Res 2008;68:10358-66.

13. Williams VC, Lucas J, Babcock MA, Gutmann DH, Korf B, Maria BL. Neurofibromatosis type 1 revisited. Pediatrics 2009;123:124-33.

14. Barbier C, Chabernaud C, Barantin L, Bertrand P, Sembely C, Sirinelli D, et al. Proton MR spectroscopic imaging of basal ganglia and thalamus in neurofibromatosis type 1: correlation with T2 hyperintensities. Neuroradiology 2011;53:141-8.

15. Moore BD, Slopis JM, Schomer D, Jackson EF, Levy BM. Neuropsychological significance of areas of high signal intensity on brain MRIs of children with neurofibromatosis. Neurology 1996;46:1660-8.

16. Goh WH, Khong PL, Leung CS, Wong VC. T2-weighted hyperintensities (unidentified bright objects) in children with neurofibromatosis 1: their impact on cognitive function. J Child Neurol 2004;19:853-8.

17. North K. Neurofibromatosis type 1. Am J Med Genet 2000;97:119-27. 